\title{
Hepatic epithelioid angiomyolipoma is a rare and potentially severe but treatable tumor: A report of three cases and review of the literature
}

\author{
WENTAO LIU ${ }^{1}$, ZIHUI MENG ${ }^{1}$, HONGYU LIU ${ }^{1}$, WEI LI ${ }^{1}$, QIONG WU ${ }^{2}$, XUEWEN ZHANG $^{1}$ and CHANGYONG E ${ }^{1}$ \\ Departments of ${ }^{1}$ Hepatobiliary and Pancreatic Surgery and ${ }^{2}$ Pathology, China-Japan Union Hospital of Jilin University, \\ Changchun, Jilin 130033, P.R. China
}

Received March 29, 2015; Accepted March 1, 2016

DOI: $10.3892 / 01.2016 .4443$

\begin{abstract}
Hepatic epithelioid angiomyolipoma (EAML) is a rare type of hepatic tumor. Due to a lack of adequate understanding about this tumor, hepatic EAML is often misdiagnosed as other diseases with similar clinical characteristics such as hepatic cancer. In the present study, 3 cases of hepatic EAML are reported, and the main clinicopathological features of this disease are presented, based on a literature search that included articles published in English between February 2000 and September 2014. A total of 24 hepatic EAML cases were considered, of which, 17 were females and 4 presented multiple liver lesions. Among the patients with single lesions, 2 underwent surgery and relapsed after 5 months and 9 years, respectively. Immunohistochemical staining was positive for human melanoma black- 45 in the present 3 cases. The aim of the present study was to focus the attention of clinicians on this type of hepatic tumor in order to improve its diagnosis and treatment.
\end{abstract}

\section{Introduction}

Epithelioid angiomyolipoma (EAML) is a rare mesenchymal neoplasia (1). At present, EAML is considered a member of the perivascular epithelioid cell (PEC) tumors known as PEComas (2), which are a type of epithelioid tumors adjacent to vessels and different from hamartomas (3). EAML is generally considered benign and the majority of patients with EAML usually have a good prognosis. However, EAML possesses malignant potential, which may lead to a poor

Correspondence to: Dr Changyong E or Professor Xuewen Zhang, Department of Hepatobiliary and Pancreatic Surgery, China-Japan Union Hospital of Jilin University, 126 Xiantai Street, Changchun, Jilin 130033, P.R. China

E-mail: edmond19811981@163.com

E-mail: zxw515@sohu.com

Key words: epithelioid angiomyolipoma, liver, hepatic cancer prognosis $(4,5)$. The treatment for patients with single lesion hepatic EAML is surgical resection (1,6-14). Multiple lesion hepatic EAML is usually metastatic, which indicates a poor prognosis of the patients. For these patients, no good treatments can be conducted. Therefore, early diagnosis of EAML is very important (15-17).

The majority of EAMLs originate in the kidneys, and primary hepatic EAML appears to be much less common than renal EAML (1). In the present study, 3 cases of hepatic EAML are presented, and a review of the relevant English literature is conducted.

\section{Case report}

Clinical data and literature review. Clinical data of the 3 EAML cases described in the present study were obtained from the records of the China-Japan Union Hospital of Jilin University (Changchun, China). The current study was approved by the ethics committee of the China-Japan Union Hospital of Jilin University.

For the literature review, different keyword combinations, including 'liver and EAML', 'liver and epithelioid angiomyolipoma', 'hepatic monotypic epithelioid angiomyolipoma' and 'atypical angiomyolipoma', were used for searching studies on hepatic EAML published in PubMed (www.ncbi.nlm.nih.gov/pubmed), MEDLINE (www.proquest.com/products-services/medline_ft.html) and Google Scholar (http://scholar.google.com). Articles were selected when full-text versions were available and contained adequate patient information for comparison. Literature reviews and duplicate reports were excluded. Table I lists the collected information, including author names and year of publication, as well as patient's age, gender, medical history, presence of single or multiple tumors, tumor site, tumor size, symptoms, treatment, results of immunohistochemical staining and follow-up (6-18). A total of 17 publications met the selection criteria, which corresponded to 24 patients, including the 3 present cases. Demographic and clinical data of the 24 patients are presented in Table I. The mean age of the patients was $47 \pm 15$ years (range, $23-80$ years). Of the 24 patients, 17 were females, and 4 exhibited multiple hepatic EAML, all patients had a history of renal EAML, 3 of which 


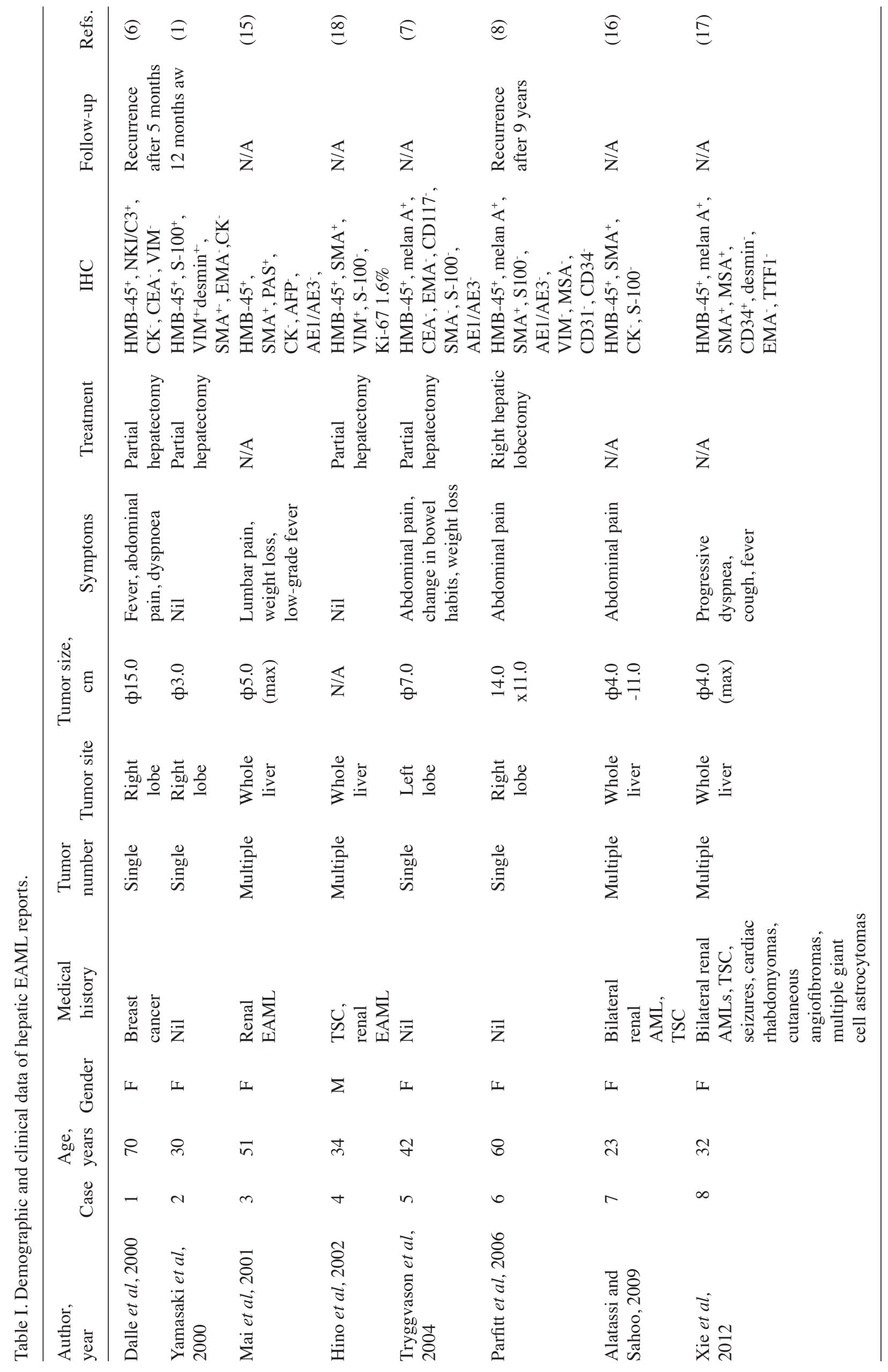




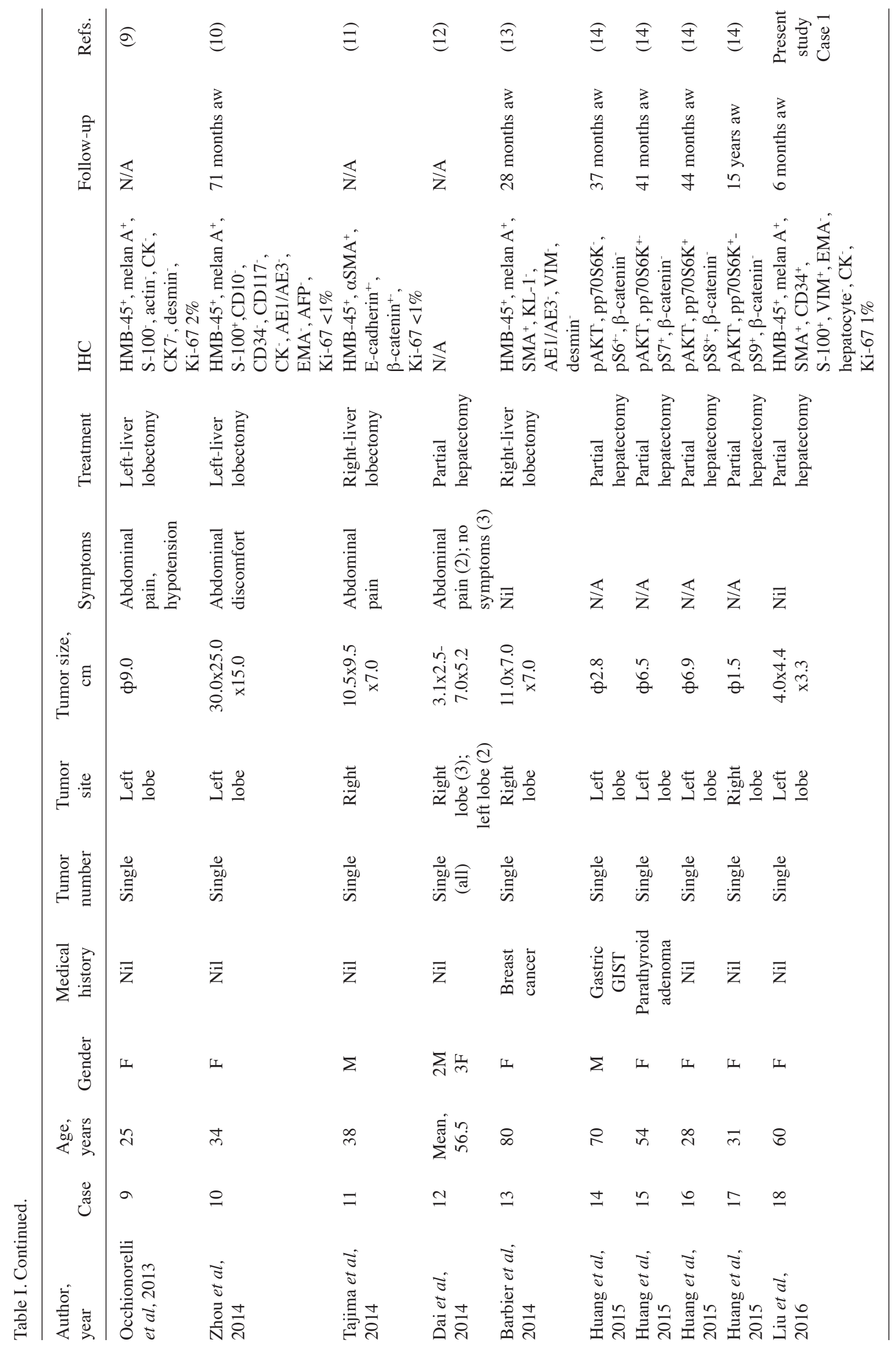




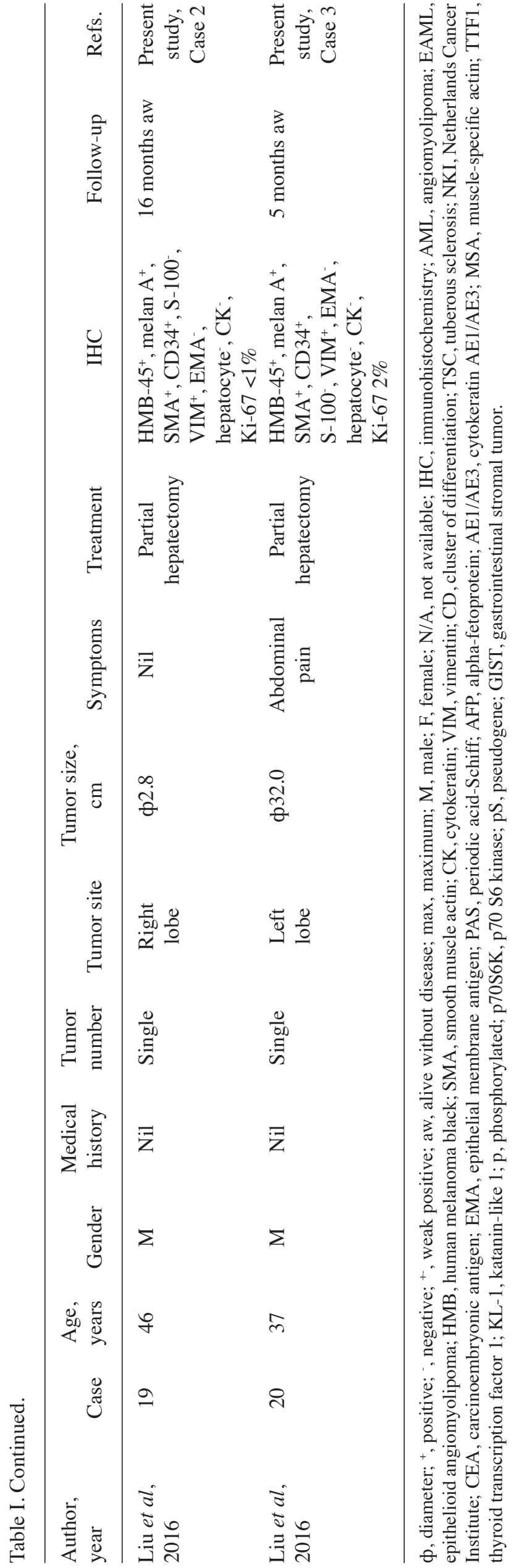

had been previously diagnosed as tuberous sclerosis (TSC). Tumor diameters of patients with single lesions varied from 2.8 to $32.0 \mathrm{~cm}$. Tumors were equally distributed between the two lobes of the liver. Of the patients with single lesions, 2 underwent surgery, and relapsed after 5 months and 9 years, respectively. All patients were positive for human melanoma black (HMB)-45 or melan A staining.

Case 1. A 60-year-old woman was admitted to the China-Japan Union Hospital of Jilin University on August 22, 2014, due to the presence of liver masses, which were noted during routine physical examination. The medical history of the patient was significant for type B hepatitis. The levels of serum alpha-fetoprotein (AFP) were normal $(3.0 \mu \mathrm{g} / \mathrm{l}$; normal range, $0-20 \mu \mathrm{g} / \mathrm{l})$. Abdominal magnetic resonance imaging (MRI; MAGNETOM Avanto 1.5; Siemens AG, Munich, Germany) revealed two hepatic masses. The mass in the right lobe was hypointense on T1-weighted images and hyperintense on T2-weighted images, which were typical features of hepatic hemangioma (3). The other mass was located in the left lobe, and was unequally isointense on T1-weighted images and mildly hyperintense on T2-weighted images (Fig. 1), thus being difficult to differentiate from hepatoma. A laparoscopic hepatic left lateral lobectomy was performed, and a neoplasia of $4 \mathrm{~cm}$ in diameter, which was protruding from the liver surface, was identified. Post-surgical pathology concluded that the tumor was a hepatic EAML. For immunohistochemistry, specimens were incubated overnight at $4^{\circ} \mathrm{C}$ with the following antibodies: Monoclonal mouse anti-human HMB-45 (\#ab787; Abcam, Cambridge, UK), monoclonal mouse anti-human melan A (\#sc-271432; Santa Cruz Biotechnology, Inc., Dallas, TX, USA), monoclonal rabbit anti-S-100 (\#ab52642; Abcam), monoclonal rabbit anti-vimentin (VIM; \#ab92547; Abcam), monoclonal mouse anti-human cluster of differentiation (CD)34 (\#sc-19587; Santa Cruz Biotechnology, Inc.), monoclonal mouse anti-pan-cytokeratin (CK; \#ab6401; Abcam), monoclonal mouse anti-human hepatocyte (\#ab75677; Abcam), polyclonal rabbit anti- $\alpha$ smooth muscle actin (SMA; \#ab5694; Abcam) and monoclonal rabbit anti-glypican-3 (\#ab124829; Abcam). All antibodies were diluted to a dilution ratio of $1: 500$ with $1 \%$ bovine serum albumin, $0.05 \%$ sodium azide and $0.01 \mathrm{M}$ phosphate-buffered saline ( $\mathrm{pH}$ 7.2). Staining demonstrated the tumor to be positive for HMB-45, melan A, S-100, SMA, VIM and CD34, but negative for CK, hepatocyte and glypican-3 (GPC-3). Ki-67 ${ }^{+}$ cells accounted for $1 \%$.

Case 2. A 46-year-old man was admitted to hospital on August 30,2013, due to a mass in the right hepatic lobe, which was noticed during routine physical examination. Viral hepatitis serology was negative and serum AFP levels were normal (5.7 $\mu \mathrm{g} / \mathrm{l}$; normal range, $0-20 \mu \mathrm{g} / \mathrm{l})$. MRI revealed a $2.8-\mathrm{cm}$ mass in the right posterior lobe, which was hypointense on T1-weighted images and hyperintense on T2-weighted images. The tumor exhibited ring-enhancements in the arterial phase, with a decrease in the portal venous/delayed phase (Fig. 1). The pathology results of an ultrasound (iU22 xMATRIX; Philips Healthcare, Andover, MA, USA)-guided fine-needle aspiration biopsy (FNAB) revealed hyperplastic lesions of pleomorphic cells. The neoplasia was removed by surgical 

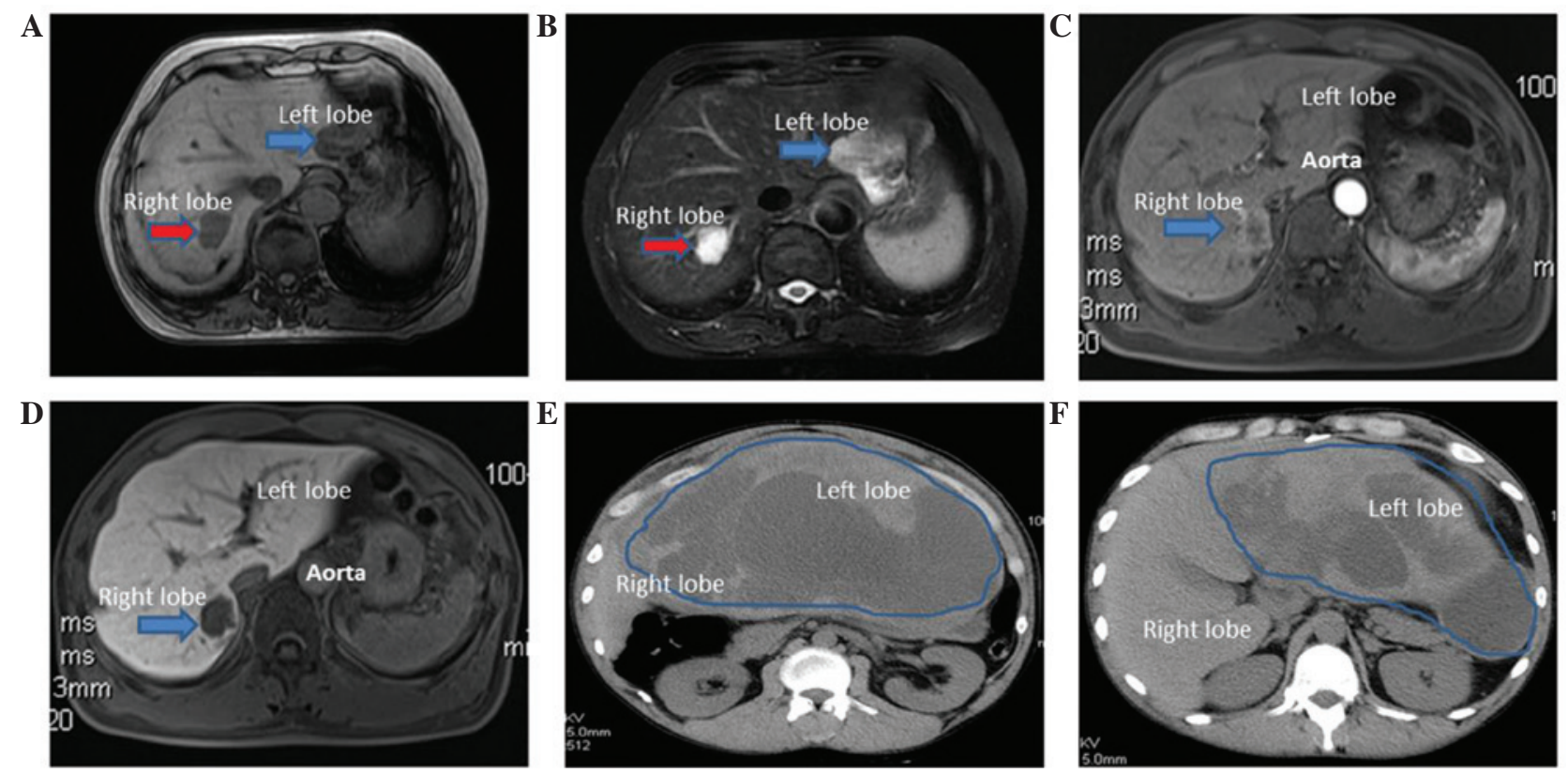

Figure 1. (A and B) MRI conducted on a 60-year-old woman with hepatic hemangioma and hepatic EAML revealed two tumors. The right lobe tumor was hypointense on T1-weighted images (A; red arrow) and hyperintense on T2-weighted images (B; red arrow), which are the typical imaging manifestations of hepatic hemangioma. The left lobe tumor displayed an unequal isointense pattern on T1-weighted images (A; blue arrow) and a mildly hyperintense signal in T2-weighted images (B; blue arrow). (C and D) MRI performed on a 46-year-old man with hepatic EAML in the right posterior lobe revealed tumor ring-enhancements in the arterial phase (C; blue arrow), with a decrease in the portal venous/delayed phase (D; blue arrow). (E and F) A CT scan performed on a 37-year-old man with a giant hepatic EAML in the left lateral lobe revealed a well-defined cystic mass located in the left lobe of the liver. These images show different scanning planes of the CT scan. The blue line circled the location of the tumor. MRI, magnetic resonance imaging; EAML, epithelioid angiomyolipoma; CT, computed tomography.

resection. Post-surgical pathology confirmed the diagnosis of hepatic EAML. For immunohistochemistry, specimens were incubated overnight at $4^{\circ} \mathrm{C}$ with the following antibodies: Monoclonal mouse anti-human HMB-45, monoclonal mouse anti-human melan A, monoclonal mouse anti-human CD34, monoclonal rabbit anti-VIM, monoclonal rabbit anti-S-100, polyclonal rabbit anti-epithelial membrane antigen (EMA; \#P15941; Abgent, Inc., San Diego, CA, USA), monoclonal mouse anti-pan-CK and monoclonal mouse anti-human hepatocyte. All antibodies were diluted with $1 \%$ bovine serum albumin, $0.05 \%$ sodium azide and $0.01 \mathrm{M}$ phosphate-buffered saline ( $\mathrm{pH}, 7.2)$. Tumor cells were positive for HMB-45, melan A, SMA, CD34 and VIM, but negative for S-100, EMA, $\mathrm{CK}$ and hepatocyte. $\mathrm{Ki}-67^{+}$cells accounted for $<1 \%$.

Case 3. A 37-year-old man presented to the emergency room on September 26, 2014, complaining of persistent abdominal pain, nausea and vomiting. Serum carbohydrate antigen 19-9 levels were elevated $(168.55 \mathrm{U} / \mathrm{ml}$; normal range, 0.00-37.00 U/ml). Abdominal contrast-enhanced computed tomography (CT; Discovery CT750 HD; GE Healthcare Bio-Sciences, Pittsburgh, PA, USA) revealed a giant hepatic tumor in the left lateral lobe. The tumor was $15.6 \times 6.3 \times 28.9 \mathrm{~cm}$ in size, and contained cystic and solid components (Fig. 1). The margins and septa of the tumor were enhanced in the arterial phase and decreased in the portal venous/delayed phase. The surgically resected specimen contained a ruptured tumor with an outflow of kermesinus fluid from the ruptured area (Fig. 2). The net weight of the tumor was $10 \mathrm{~kg}$ and its diameter was $32.0 \mathrm{~cm}$. Pathology confirmed the diagnosis of hepatic EAML. Part of the tumor tissue was necrotic. For immuno-

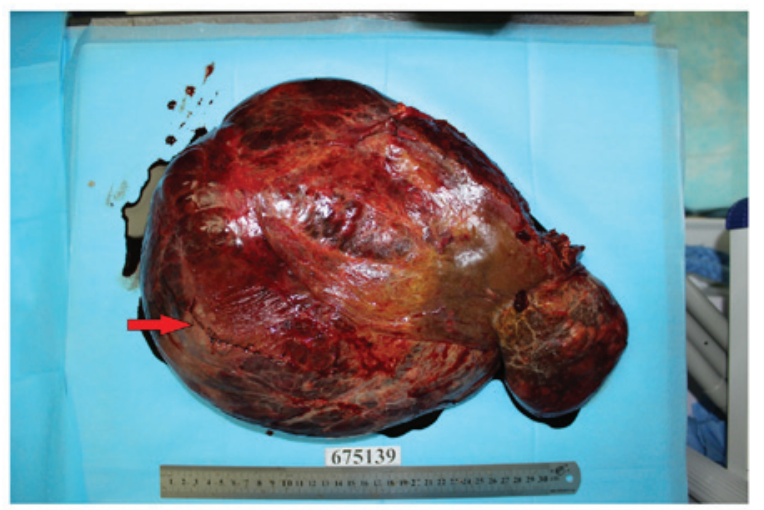

Figure 2. Surgical specimen of partial hepatectomy for a 32-cm epithelioid angiomyolipoma. The red arrow indicates the ruptured area.

histochemistry, specimens were incubated overnight at $4^{\circ} \mathrm{C}$ with the following antibodies: Monoclonal mouse anti-human HMB-45, monoclonal mouse anti-human melan A, monoclonal mouse anti-human CD34, monoclonal rabbit anti-S-100, polyclonal rabbit anti-EMA (\#P15941; Abgent, Inc., San Diego, CA, USA), polyclonal rabbit anti-human AFP (\#ab182645; Abcam), monoclonal mouse anti-pan-CK, monoclonal mouse anti-human hepatocyte, polyclonal rabbit anti-chromogranin (\#P10645; Abgent, Inc.) and polyclonal rabbit anti-synaptophysin (\#ab14692; Abcam). All antibodies were diluted with $1 \%$ bovine serum albumin, $0.05 \%$ sodium azide and $0.01 \mathrm{M}$ phosphate-buffered saline $(\mathrm{pH}, 7.2)$. All antibodies were diluted with $1 \%$ bovine serum albumin. $0.05 \%$ sodium azide and $0.01 \mathrm{M}$ phosphate-buffered saline (pH, 7.2). Staining was 

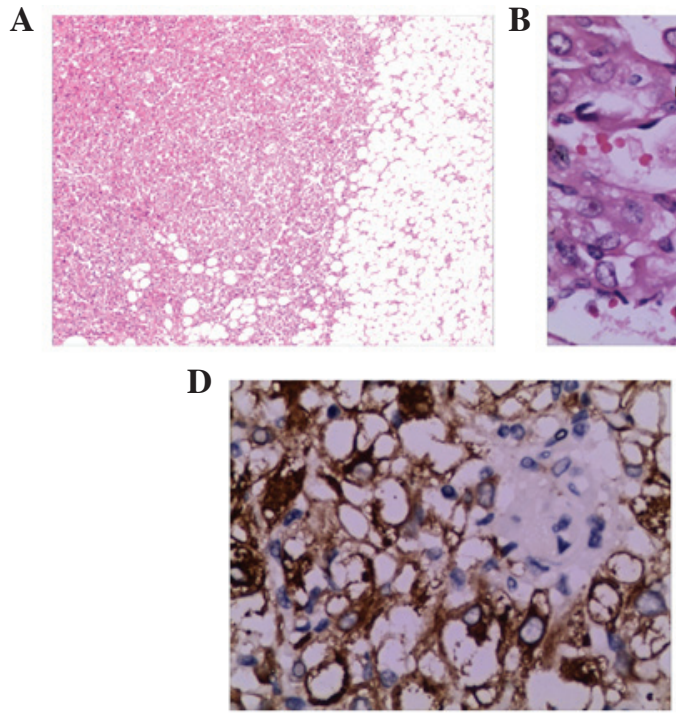
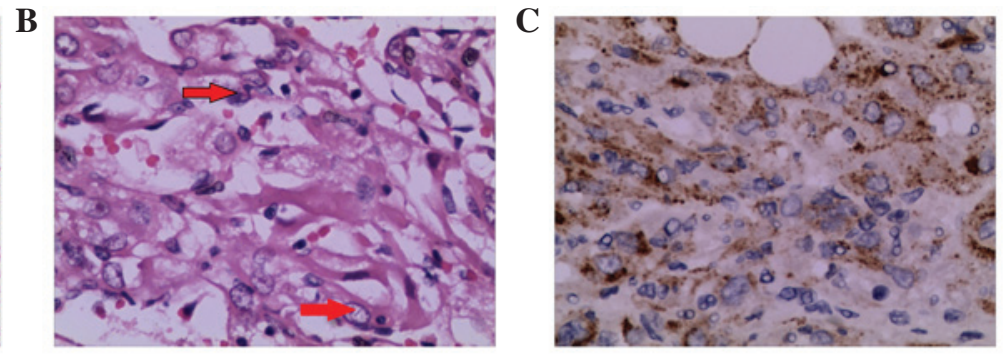

$\mathbf{E}$

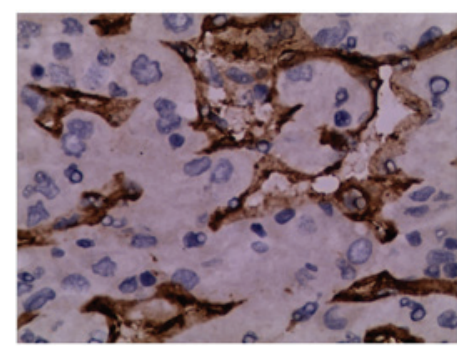

Figure 3. Histological features of hepatic EAML (case 3). (A) Histological features of EAML, as revealed by H\&E staining; magnification, x40. (B) Epithelioid cells (indicated by red arrows) were rounded or polygonal with abundant cytoplasm (H\&E staining; magnification, $\mathrm{x} 400$ ). (C) Tumor cells were strongly and diffusely positive for human melanoma black-45 (magnification, x400). (D) Tumor cells were strongly positive for melan A (magnification, x400). (E) The tumor was positive for the vascular marker cluster of differentiation 34 (magnification, x400). EAML, epithelioid angiomyolipoma; H\&E, hematoxylin and eosin.

positive for HMB-45, melan A, SMA and CD34, but negative for S-100, EMA, AFP, CK, hepatocyte, GPC-3, chromogranin and synaptophysin (Fig. 3). Ki-67+ cells accounted for $2 \%$.

\section{Discussion}

In 2002, the World Health Organization recognized PEComas as a group of neoplasms with PEC differentiation (19). PEComas include AML, lymphangioleiomyomatosis and clear cell 'sugar' tumor (19). EAML is a type of AML composed almost exclusively of epithelioid cells with pronounced abnormal blood vessels and few or no lipocytes (20). One of the criteria for EAML in the kidney is that epithelioid cells occupy $>10 \%$ of the tumor (21).

EAML mostly occurs in the kidney, although in rare cases, it develops in the liver, which is known as hepatic EAML (22). Hepatic EAML mostly affects females (male to female ratio, $\sim 0.4$ ). The majority of hepatic tumors reported in the literature are single lesions (1,6-14). In total, 4 of the patients identified with hepatic EAML in the current literature review presented multiple lesions, and all of them had a history of renal EAML. Therefore, it is very likely that their hepatic tumors corresponded to metastatic lesions that originated in the kidneys. In addition, 3 of these patients had been diagnosed as TSC with loss of heterozygosity at TSC1 (9q34) and TSC2 (16p13), which suggests that EAML may be associated with those genes (23).

Usually, patients with hepatic EAML are clinically asymptomatic when the tumors are small $(13,14)$. However, when the tumors are very large, patients may present with abdominal distension and pain $(7,8,16)$. According to the present literature review and the 3 cases reported in the current study, a tumor measuring $>5 \mathrm{~cm}$ in diameter may be associated with abdominal pain, fever, weight loss and changes in bowel habits (9). The tumor diameter observed in case $3(32.0 \mathrm{~cm})$ was the largest reported thus far (10). Tumor size is also an important factor for predicting tumor rupture (9). To the best of our knowledge, the patient of case 3 is the 7 th case of hepatic AML rupture that has been reported in the literature to date (11).

Imaging features of hepatic EAML vary from case to case and may lack specificity (24). Usually, the imaging features of the tumors are associated with histological components (24). Thus, the majority of reported hepatic EAML tumors were completely devoid of adipose tissue, and fat attenuation was rarely observed in CT or MRI images (24). By contrast, nearly all tumors were markedly enhanced in the arterial phase, indicating that hepatic EAML is a hypervascularized tumor (7). There are two types of enhancement patterns in the portal venous/delayed phase (25): Lesions with abundant central vessels exhibited a rapid contrast decrease, whereas lesions with small or no vessels demonstrated prolonged enhancement (26). The majority of lesions exhibited a significantly reduced contrast in the portal venous/delayed phase (24). Accordingly, the tumor in case 2 revealed ring-enhancements in the arterial phase with a decrease in the portal venous/delayed phase, while the margins and septa of the tumor in case 3 were enhanced in the arterial phase and decreased in the portal venous/delayed phase.

Immunohistochemistry is one of the most important diagnostic tools for hepatic EAML $(7,15)$. This type of tumor usually displays immunoreactivity for both melanocytic (HMB-45 and melan A) and myoid (SMA and muscle-specific actin) markers (27). All the 3 cases described in the present report were positive for HMB-45, melan and SMA, but negative for hepatocyte and CK. Thus, FNAB appears to be important for diagnosing hepatic EAML prior to surgery (17).

In conclusion, surgical resection is the first therapeutic option for primary hepatic EAML, which should be conducted as early as possible, due to the risks of progressive increase and eventual rupture of the tumor. Furthermore, hepatic EAML has a metastasis potential, particularly in patients with a prior medical history of TSC. The responses of neoplastic hepatic 
EAML to conventional chemotherapy and radiotherapy remain poorly documented, and required to be evaluated by further clinical trials.

\section{References}

1. Yamasaki S, Tanaka S, Fujii H, Matsumoto T, Okuda C, Watanabe G and Suda K: Monotypic epithelioid angiomyolipoma of the liver. Histopathology 36: 451-456, 2000.

2. Martignoni G, Pea M, Reghellin D, Zamboni G and Bonetti F: PEComas: The past, the present and the future. Virchows Arch 452: 119-132, 2008.

3. Brown MA and Semelka RC (eds): MRI Basic Principles and Applications. 3rd edition. John Wiley \& Sons, Inc., Hoboken, NJ, 2003.

4. Harris GC, McCulloch TA, Perks G and Fisher C: Malignant perivascular epithelioid cell tumour ("PEComa") of soft tissue: A unique case. Am J Surg Pathol 28: 1655-1658, 2004.

5. Lehman NL: Malignant PEComa of the skull base. Am J Surg Pathol 28: 1230-1232, 2004.

6. Dalle I, Sciot R, de Vos R, Aerts R, van Damme B, Desmet V and Roskams T: Malignant angiomyolipoma of the liver: A hitherto unreported variant. Histopathology 36: 443-450, 2000.

7. Tryggvason G, Blöndal S, Goldin RD, Albrechtsen J, Björnsson J and Jónasson JG: Epithelioid angiomyolipoma of the liver: Case report and review of the literature. APMIS 112: 612-616, 2004

8. Parfitt JR, Bella AJ, Izawa JI and Wehrli BM: Malignant neoplasm of perivascular epithelioid cells of the liver. Arch Pathol Lab Med 130: 1219-1222, 2006.

9. Occhionorelli S, Dellachiesa L, Stano R, Cappellari L, Tartarini D, Severi S, Palini GM, Pansini GC and Vasquez G: Spontaneous rupture of a hepatic epithelioid angiomyolipoma: Damage control surgery. A case report. G Chir 34: 320-322, 2013

10. Zhou Y, Chen F, Jiang W, Meng Q and Wang F: Hepatic epithelioid angiomyolipoma with an unusual pathologic appearance: Expanding the morphologic spectrum. Int J Clin Exp Pathol 7 6364-6369, 2014.

11. Tajima S, Suzuki A and Suzumura K: Ruptured hepatic epithelioid angiomyolipoma: A case report and literature review. Case Rep Oncol 7: 369-375, 2014.

12. Dai CL, Xue LP and Li YM: Multi-slice computed tomography manifestations of hepatic epithelioid angiomyolipoma. World J Gastroenterol 20: 3364-3368, 2014.
13. Barbier L, Torrents $\mathbf{J}$ and Hardwigsen J: Hepatic angiomyolipoma: What management? Acta Chir Belg 114: 139-142, 2014.

14. Huang SC, Chuang HC, Chen TD, Chi CL, Ng KF, Yeh TS and Chen TC: Alterations of the mTOR pathway in hepatic angiomyolipoma with emphasis on the epithelioid variant and loss of heterogeneity of TSC1/TSC2. Histopathology 66: 695-705, 2015.

15. Mai KT, Yazdi HM, Perkins DG and Thijssen A: Fine needle aspiration biopsy of epithelioid angiomyolipoma. A case report. Acta Cytol 45: 233-236, 2001.

16. Alatassi $\mathrm{H}$ and Sahoo S: Epithelioid angiomyolipoma of the liver with striking giant cell component: Fine-needle aspiration biopsy findings of a rare neoplasm. Diagn Cytopathol 37: 192-194, 2009.

17. Xie L, Jessurun J, Manivel JC and Pambuccian SE: Hepatic epithelioid angiomyolipoma with trabecular growth pattern: A mimic of hepatocellular carcinoma on fine needle aspiration cytology. Diagn Cytopathol 40: 639-650, 2012.

18. Hino A, Hirokawa M, Takamura K and Sano T: Imprint cytology of epithelioid angiomyolipoma in a patient with tuberous sclerosis. A case report. Acta Cytol 46: 545-549, 2002.

19. Hornick JL and Fletcher CD: PEComa: What do we know so far? Histopathology 48: 75-82, 2006.

20. Mai KT, Perkins DG and Collins JP: Epithelioid cell variant of renal angiomyolipoma. Histopathology 28: 277-280, 1996.

21. Aydin H, Magi-Galluzzi C, Lane BR, Sercia L, Lopez JI, Rini BI and Zhou M: Renal angiomyolipoma: Clinicopathologic study of 194 cases with emphasis on the epithelioid histology and tuberous sclerosis association. Am J Surg Pathol 33: 289-297, 2009.

22. Eble JN: Angiomyolipoma of kidney. Semin Diagn Pathol 15: 21-40, 1998.

23. Ooi SM, Vivian JB and Cohen RJ: The use of the Ki-67 marker in the pathological diagnosis of the epithelioid variant of renal angiomyolipoma. Int Urol Nephrol 41: 559-565, 2009.

24. Ji JS, Lu CY, Wang ZF, Xu M and Song JJ: Epithelioid angiomyolipoma of the liver: CT and MRI features. Abdom Imaging 38: 309-314, 2013.

25. Xu PJ, Shan Y, Yan FH, Ji Y, Ding Y and Zhou ML: Epithelioid angiomyolipoma of the liver: Cross-sectional imaging findings of 10 immunohistochemically-verified cases. World J Gastroenterol 15: 4576-4581, 2009.

26. Xiao W, Zhou M, Lou H, Wang Z and Zhang M: Hemodynamic characterization of hepatic angiomyolipoma with least amount of fat evaluated by contrast-enhanced magnetic resonance angiography. Abdom Imaging 35: 203-207, 2010.

27. Park HK, Zhang S, Wong MK and Kim HL: Clinical presentation of epithelioid angiomyolipoma. Int J Urol 14: 21-25, 2007. 